\title{
Involvement of gelsolin in TGF-beta 1 induced epithelial to mesenchymal transition in breast cancer cells
}

\author{
Zhi-Yuan Chen ${ }^{1 \dagger}$, Pei-Wen Wang ${ }^{2 \dagger}$, Dar-Bin Shieh ${ }^{2,3+}$, Kuan-Ying Chiu ${ }^{1}$ and Ying-Ming Liou ${ }^{1,4^{*}}$
}

\begin{abstract}
Background: Increasing evidence suggests that transforming growth factor-beta 1 (TGF- $\beta 1$ ) triggers epithelial to mesenchymal transition (EMT) and facilitates breast cancer stem cell differentiation. Gelsolin (GSN) is a ubiquitous actin filament-severing protein. However, the relationship between the expression level of GSN and the TGF- $\beta$ signaling for EMT progression in breast cancer cells is not clear.
\end{abstract}

Results: TGF- $\beta 1$ acted on MDA-MB231 breast cancer cells by decreasing cell proliferation, changing cell morphology to a fibroblast-like shape, increasing expressions for CD44 and GSN, and increasing EMT expression and cell migration/ invasion. Study with GSN overexpression (GSN op) in both MDA-MB231 and MCF-7 cells demonstrated that increased GSN expression resulted in alterations of cell proliferation and cell cycle progression, modification of the actin filament assembly associated with altering cell surface elasticity and cell detachment in these breast cancer cells. In addition, increased cell migration was found in GSN op MDA-MB231 cells. Studies with GSN op and silencing by small interfering RNA verified that GSN could modulate the expression of vimentin. Sorted by flow cytometry, TGF- $\beta 1$ increased subpopulation of CD44+/CD22- cells increasing their expressions for GSN, Nanog, Sox2, Oct4, N-cadherin, and vimentin but decreasing the E-cadherin expression. Methylation specific PCR analysis revealed that TGF- $\beta 1$ decreased $50 \%$ methylation but increased 3-fold unmethylation on the GSN promoter in CD44+/CD22- cells. Two DNA methyltransferases, DNMT1and DNMT3B were also inhibited by TGF- $\beta 1$.

Conclusions: TGF- $\beta 1$ induced epigenetic modification of GSN could alter the EMT process in breast cancer cells.

Keywords: GSN, TGF- $\beta 1$, EMT, Methylation specific PCR, DNA methyltransferases

\section{Background}

TGF- $\beta 1$ is a secreted cytokine involved in controlling gene expression and ultimately cell cycle and tissue repair [1]. In the initial stage of tumorigenesis, TGF- $\beta 1$ acts as a tumor suppressor [2]. With tumor progression cancer cells overproducing TGF- $\beta 1$ turn to promote cancer cell proliferation, invasion and metastasis, hence become resistant to the TGF- $\beta 1$-induced growth inhibition in their later stage [2]. In addition, the TGF- $\beta 1$ signaling pathway has been shown to cause a constitutive

\footnotetext{
* Correspondence: ymlion@dragon.nchu.edu.tw

${ }^{\dagger}$ Equal contributors

'Department of Life Sciences, National Chung-Hsing University, Taichung 40227, Taiwan

${ }^{4}$ Rong Hsing Research Center for Translational Medicine, National Chung Hsing University, Taichung 40227, Taiwan

Full list of author information is available at the end of the article
}

epithelial to mesenchymal transition (EMT) facilitating a highly invasive and metastatic phenotype in breast tumors [3, 4]. Recent evidence also demonstrated that TGF- $\beta$ could increase breast tumor-initiating cell numbers in the low claudin expression subtype of breast tumors [5]. Apparently, the TGF- $\beta$ signaling for EMT, cell motility, and invasiveness might play an important role in enriching the cancer stem cell (CSC) pool in breast tumors [6].

The actin cytoskeleton underlies several cellular functions including cell differentiation in both normal and tumor cells [7-10]. It has been shown that the TGF- $\beta$ signaling via Smad and p38MAPK caused upregulation of actin binding proteins, including tropomyosin, $\alpha$-actinin, and calponin, to control the stress fiber formation, which might contribute to modulation of cell motility and invasive phenotype with EMT in tumor cells [11, 12]. Gelsolin 
(GSN), one of the most potent members of the actinsevering superfamily, plays a key role in the regulation of actin filament assembly and disassembly $[13,14]$. GSN involves in many cellular properties for carcinogenesis phenotypes, EMT, motility, apoptosis, proliferation, and differentiation [15-17]. However, it remains to be determined if the TGF- $\beta$ signaling events also include the modulation of GSN expression for promotion of breast cancer cell differentiation.

Similar to leukemia, several CSC-like subpopulations have been thought to exist in breast cancers $[18,19]$. These breast CSCs acquire the ability to differentiate into all the different cells found within a tumor that become chemotherapy resistant $[20,21]$. In this study, the TGF$\beta 1$-induced MDA-MB231breast cancer cells as a model for CSC differentiation were used to investigate whether the expression level of GSN is regulated by the TGF- $\beta 1$ signaling for promoting breast CSC differentiation. Results reported here suggest that GSN involves in the TGF- $\beta 1$ driving CSC differentiation by the process of EMT in breast cancer cells.

\section{Methods}

\section{Cell culture and TGF- $\beta 1$ treatment}

MCF-7 cells in Dulbecco's modified essential medium (DMEM, Gibco) containing glutamine, while MDAMB231 in DMEM containing sodium bicarbonate, both supplemented with antibiotics and $10 \%$ fetal bovine serum (FBS) were cultured in a $5 \% \mathrm{CO}_{2}$ incubator at $37^{\circ} \mathrm{C}$. To determine the growth inhibition, 5000 cells for each cell line were plated in 96-well plates with or without TGF- $\beta 1$ treatment. Cell viability and proliferation was measured using the MTT [3-(4,5-dimethylthiazol2-yl)-2,5-diphenyltetrazolium bromide] assay (ATCC, Manassas, VA, USA) [22, 23].

\section{Cell migration and invasion assay}

The invasion and migration assay of cancer cells were performed using modified Boyden chamber assay with a FalconTM Cell Culture Insert (BD Biosciences). To create an invasion assay the membrane was coated with a Matrigel to simulate the typical matrices that cancer cells encounter during the invasion process in vivo. In contrast, the membrane without coating was used only for the migration assay. In both measurements, the cells $\left(10^{5}\right.$ cells $\left./ \mathrm{ml}\right)$ were placed on upper side and a chemoattractant (10\% FBS) on the lower side. Cells that migrated through the membrane were fixed with $100 \%$ absolute alcohol, stained with crystal violet. After air dried, migrated cells were then added with $30 \%$ acetic acid, and quantitated by measuring the optical density at $590 \mathrm{~nm}$ in a micro-plate reader.

\section{Cell cycle phase determination}

Cells $\left(10^{7}\right)$ were seeded in a $10-\mathrm{cm}$ dish in DMEM-0.2 \% FBS and cultured in a $\mathrm{CO}_{2}$ incubator at $37{ }^{\circ} \mathrm{C}$ for $24 \mathrm{~h}$. The cells were then changed to fresh medium, trypsinized, and centrifuged. The pellet was washed and re-suspended in $1 \mathrm{ml}$ of pre-chilled phosphate buffer solution (PBS) and the cells fixed by gradually adding $3 \mathrm{ml}$ of $95 \%$ ethanol, then were stored in a deep freezer $\left(-20^{\circ} \mathrm{C}\right)$ overnight. The cells were then washed three times by centrifugation and resuspension in pre-chilled PBS. To stain the cells with propidium iodide (PI), the cells were resuspended in PBS containing $0.1 \%$ Triton X-100, $20 \mu \mathrm{g} / \mathrm{ml}$ of PI, and $0.2 \mathrm{mg} / \mathrm{ml}$ of RNase A and incubated for $30 \mathrm{~min}$ at room temperature in the dark. Samples were analyzed on a flow cytometer (FC500 Flow Cytometry System, Beckman Coulter, Inc.) with a $488 \mathrm{~nm}$ excitation laser. The cell cycle phases were determined using the computerized software provided with the machine (CXP Software, Beckman Coulter, Inc.).

\section{Cell staining for FACS flow cytometry}

Cells $\left(10^{7}\right)$ were incubated with fluorochrome-conjugated antibodies followed by fluorescence-activated cell sorting (FACS). To characterize stem cell markers in breast cancer cells, the following antibodies were used: allophycocyanin (APC)-conjugated anti-human CD44 (clone G44-26, mouse IgG2b, BD Pharmingen, CA, USA), phycoerythrin (PE)-conjugated anti-human CD24 (clone ML5, mouse IgG2a, BD Pharmingen, CA, USA). Single-cell suspensions dissociated from the dishes, using cell dissociation buffer (GIBCO), were stained with flurochrome antibody for $30 \mathrm{~min}$ at $4{ }^{\circ} \mathrm{C}$ and analyzed by a flow cytometry of $\mathrm{BD}$ FACS Aria apparatus (BD Biosciences, Palo Alto, CA).

\section{RNA extraction, semi-quantitative RT-PCR, real-time qPCR, comparative $\mathrm{CT}$ method for quantification of $\mathrm{mRNA}$ expression}

The procedures for RNA extraction, semi-quantitative reverse transcription polymerization chain reaction (semiquantitative RT-PCR), and qPCR were described previously [22-24]. SYBR Green dye was used as a real-time reporter of the presence of double-stranded DNA. The following primers specific for stem cell markers (i.e. Oct4, Sox2 and Nanog), for EMT markers (i.e. N-cadherin, and vimentin, and E-cadherin), for GSN, and for DNMT1/ DNMT3B were synthesized: Oct4, forward 5 '-CCTGAAG CAGAAGAGGATCA-3' and reverse 5'-CCGCAGCTT ACACATGTTCT-3'; Sox2, forward 5' -CGATGCCGACA AGAAAACTT $-3^{\prime}$ and reverse $5^{\prime}$-CAAACTTCCTGCAA AGCTCC-3'; Nanog, forward 5'-TTCAGTCTGGACAC TGGCTG-3' and reverse $5^{\prime}$-CTCGCTGATTAGGCTC CAAC-3'; E-cadherin, forward 5' -GCCTCCTGAAAAGA GAGTGGAAG-3' and reverse 5'-TGGCAGTGTCTCTC CAAATCCG-3'; N-cadherin, forward 5'-ACAGTGGCC 
ACCTACAAAGG-3' and reverse 5' -CCGAGATGGGGT TGATAATG-3'; Vimentin, forward 5' -AGGAAATGGCT CGTCACCTTCGTGAATA-3' and reverse 5'-GGAGTG TCGGTTGTTAAGAACTAGAGCT-3'; GSN, forward 5' -ACGGACCCAGCCAATCG-3' and reverse 5'-CATC ATCCCAGCCAAGGAA-3'; DNMT1, forward 5' -AAGA CAAAGACCAGGATGAGAAG-3' and reverse 5'-GGGT GTTGGTTCTTTGGTTTG-3'; DNMT3B, forward 5'-C CATTCGAGTCCTGTCATTG-3' and reverse 5'-GCAA TGGACTCCTCACACAC-3'. The primers for actin binding proteins were: Tropomyosin 1 (Tm1), forward $5^{\prime}-\mathrm{TC}$ ATCATTGAGAGCGACCTG-3' and reverse $5^{\prime}$-CTTGT CGGAAAGGACCTTGA-3'; Caldesmon, forward 5'-CT GGCTTGAAGGTAGGGGTTT $-3{ }^{\prime}$ and reverse $5^{\prime}$-TTG GGAGCAGGTGACTTGTTT-3'; Profilin, forward 5 ' -CT GTCAGGACGCGGCCATCG-3' and reverse 5' -AACGT TTTCCCGGGGACGGC-3'. GAPDH, an internal control, had the forward primer 5 '-ATGGGGAAGGTGAAGGTC G-3' and the reverse primer 5'-TAAAAGCAGCCCTGG TGACC-3', respectively.

\section{Immunoblotting}

Protein contents of total cell lysates from TGF- $\beta 1$ treated or untreated cells were analyzed by western blot. Samples with same amounts of protein were separated by sodium dodecyl sulfate-polyacrylamide gel electrophoresis, then the proteins were electro-transferred onto polyvinylidene difluoride membranes. The primary antibodies used were: mouse monoclonal anti-human GSN (Sigma GS-2C4; 1:10000 dilution), mouse monoclonal anti-human CD44 (Abcam;1:1000 dilution), mouse monoclonal anti-human E-cadherin (2Q663) (sc-71008), human- $\beta$-catenin (9 F2) (sc-47752), human GSK-3 $\beta$ (H-76) (sc-9166), human cyclin D1 (DSC-6) (sc-20044), mouse monoclonal antihuman N-cadherin (H-63) (sc-7939) (all from Santa Cruz; 1: 2000 dilution), mouse monoclonal anti- $\beta$-actin (Sigma, 1: 10000 dilution), rabbit monoclonal anti-Tm1 (Sigma, 1:2000 dilution), rabbit monoclonal anti-caldesmon (Santa Cruz, 1:5000 dilution), rabbit monoclonal anti-profilin (Santa Cruz, 1:3000 dilution), rabbit monoclonal antihuman vimentin (Abcam; 1:1000 dilution), and rabbit polyclonal anti-GAPDH (GeneTex GTX100118; 1:5000). The secondary antibodies used (1:5000 dilution) were goat anti-rabbit IgG (Sigma), and goat anti-mouse IgG (Sigma).

\section{GSN overexpression and silencing by small interfering RNA (siRNA)}

The pc6-GSN plasmid construct was cotransfected with GSN into both MCF-7 and MDA-MB231 breast cancer cells using lipofectamine 2000 (Invitrogen). The fulllength cytoplasmic GSN cDNA [24, 25] was cloned into the expression vector pcDNA6-V5/His. Before transfection, cells were cultured in a 6-well plate containing culture medium without antibiotics at a density of 70-80 \% confluence. Both the lipofectamine and DNA constructs were diluted with transfection medium without serum and incubated for $5 \mathrm{~min}$. Subsequently, the diluted DNA constructs and diluted lipofectamine were mixed at a 1:2.5 ratio of DNA to lipofectamine. After gentle shaking and incubation for $20 \mathrm{~min}$, the DNA-lipofectamine complexes were added to each well and incubated in a $\mathrm{CO}_{2}$ incubator at $37{ }^{\circ} \mathrm{C}$ for $6 \mathrm{~h}$. The culture medium was replaced with serum-containing DMEM.

Sixty to eighty percent confluent cells were transfected with siRNAs directed to human GSN (sc-7330), according to the manufacturer's guidelines (Santa Cruz Biotechnology). The cells received $10 \mu \mathrm{M}$ siRNA were incubated for $6 \mathrm{~h}$ at $37^{\circ} \mathrm{C}$ in a $\mathrm{CO}_{2}$ incubator. At 24 and $48 \mathrm{~h}$ after transfection total RNA were extracted for reverse transcription and GPCR measurements to confirm downregulation of GSN expression [22, 24].

\section{Atomic force microscopy}

DI-Dimension 3100 AFM (Digital Instruments, Santa Barbara, CA) was applied to obtain cell surface contour images in contact mode and measure the interfacial forces in tapping mode [24]. The V-shaped silicon cantilevers with a spring constant of $\sim 0.9$ to $0.12 \mathrm{~N} / \mathrm{m}$ were used for imaging cell surface areas $(20 \times 20 \mu \mathrm{m})$ in phosphate buffer. Approximately $10-20$ spots of this scanning region were randomly selected using the same probe to extend forward $1 \mathrm{~nm}$ deep and to retract back to the starting point. The retracting force-distance curves were used to calculate the adhesion forces that correspond to the elasticity of cell membrane surface.

\section{Cell detachment measurements}

Cells $\left(10^{5}\right)$ plated on a 12-well plate were treated with $150 \mu \mathrm{l}$ of TrypLETM (Invitrogen) for 60, 90, and $180 \mathrm{~s}$, respectively, followed by adding $1 \mathrm{ml}$ of cell culture medium to stop the action of trypsin. Detached cells post treatments with TrypLETM for 60, 90, and $180 \mathrm{~s}$ were collected and measured the cell number by trypan blue exclusion assay. The detached cell numbers for the group with $180 \mathrm{~s}$ post trypsin treatment were used to normalize the degree of cell detachment for the group with 60 or $90 \mathrm{~s}$ after trypsin treatments.

\section{Methylation-specific PCR analyses}

Methylation status of GSN was determined by methylation-specific PCR (MSPCR) using bisulphatemodified genomic DNA as the template. Genomic DNA was treated with bisulphate by using the Zymo DNA Modification Kit (Zymo Rearch, Orange, CA, USA) according the protocol provided by the manufacturer. Methylation-specific GSN primers are: forward: 5'-ATGT TTATTTGATAAACGAGGGAAAC-3', and reverse: 5'-C ATTAAACAAACGCCTCGAA-3'; and unmethylation- 
specific GSN primers: forward: 5'-GTTTATTTGATAAAT GAGGAAATGG-3' and reverse: 5'TAAACCATTAAAC ACCTCAAA-3'.

\section{Statistical analysis}

Quantitative values are presented as the mean and standard error of the mean (mean \pm SEM). A difference was considered to be statistically significant when the $\mathrm{P}$ value was less than 0.05 .

\section{Results}

Effects of TGF- $\beta 1$ treatment on cell proliferation, the expression of CD44, GSN, and EMT markers (i.e. $\mathrm{N}$-cadherin, vimentin, and E-cadherin) in MDA-MB231 breast cancer cells To test the appropriate condition for TGF- $\beta 1$ induction, MDA-MB231 breast cancer cells were treated with TGF- $\beta 1$ from 1 to $20 \mathrm{ng} / \mathrm{ml}$ for $0,24,48,72$, and $96 \mathrm{~h}$. Treatment with $>1 \mathrm{ng} / \mathrm{ml}$ TGF- $\beta 1$ for $72 \mathrm{~h}$ sufficiently decreased cell proliferation (Fig. 1a) in MDA-MB231 cells. Applying different concentrations $(1,2,5,10,20 \mathrm{ng} / \mathrm{ml})$ of TGF- $\beta 1$ to cells in culture medium for 3 days, MDA-MB231 cells showed a dose dependent increase in protein expressions for CD44 and GSN (Fig. 1b). In addition, MDA-MB231 cells treated with TGF- $\beta 1$ from 1 to $5 \mathrm{ng} / \mathrm{ml}$ for 3 days also increased the expression of mesenchymal cell markers (i. e. $\mathrm{N}$-cadherin, vimentin) but decreased the expression for epithelial cell marker (i.e. E-cadherin) (Fig. 1c). In parallel, the mRNA level was increased for GSN and N-cadherin and vimentin but decreased for E-cadherin in MDAMB231 cells treated with $2 \mathrm{ng} / \mathrm{ml}$ TGF- $\beta 1$ for 3 days, as compared to control without TGF- $\beta 1$ (Fig. 1d). In addition, the TGF- $\beta 1$ treatment was confirmed to facilitate cell migration and invasion in MDA-MB231 breast cancer cells.
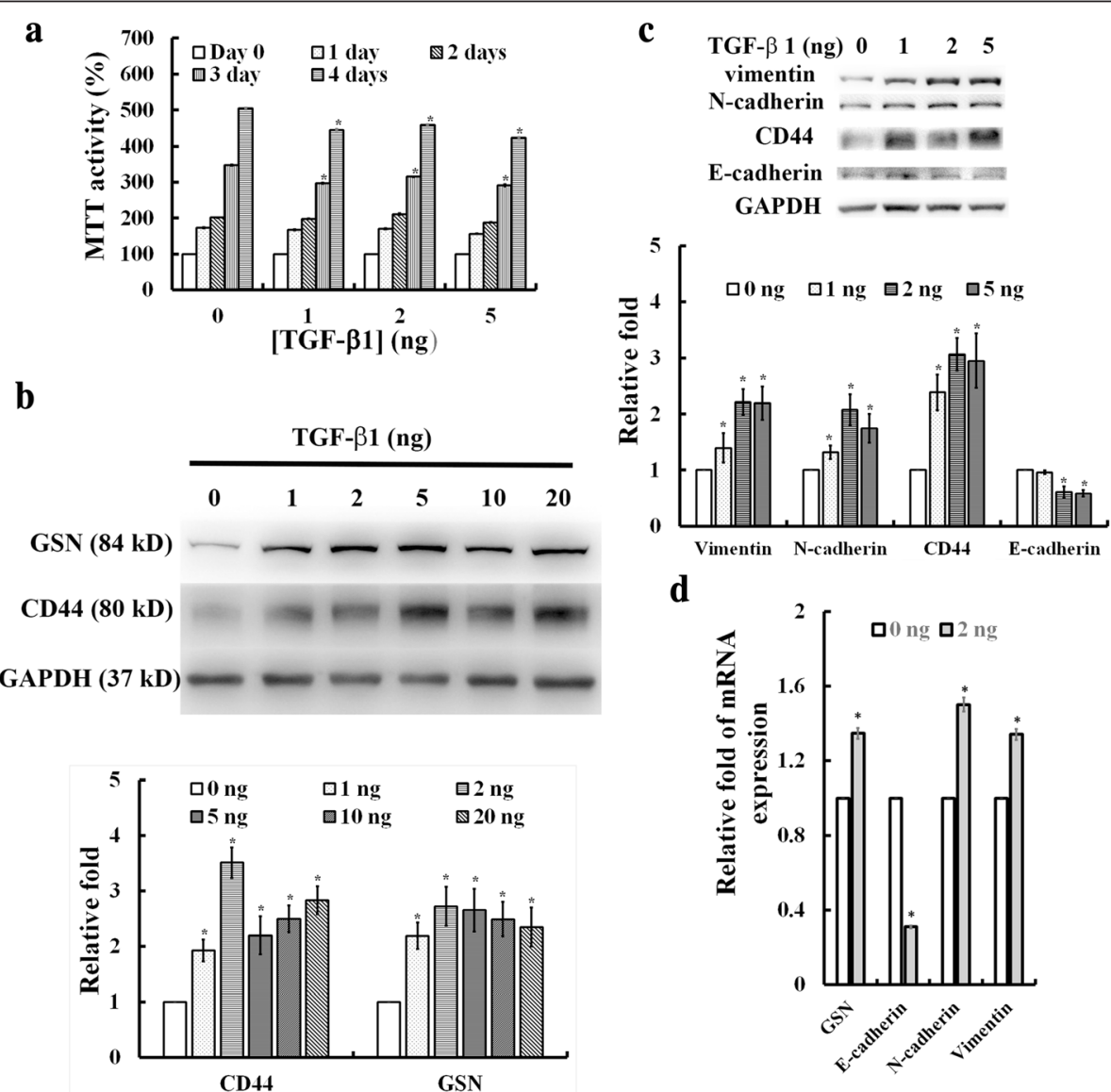

Fig. 1 Effects of TGF- $\beta 1$ treatment on cell proliferation, the expression of CD44, GSN, and EMT markers (i.e. N-cadherin, vimentin, and E-cadherin) in MDA-MB231 breast cancer cells. a MTT assay of cell viability after incubation of MDA-MB231 breast cancer cells with TGF- $\beta 1$ from 1 to 5 ng/ml for $0,24,48,72$, and 96 h. b Western blotting (top) with quantitative analyses (bottom) showed a dose dependent increase in protein expressions for CD44 and GSN in MDA-MB231 cells treated with 0, 1, 2, 5, 10, $20 \mathrm{ng} / \mathrm{ml}$ of TGF- $\beta 1$ for $72 \mathrm{~h}$. c Western blotting (top) with quantitative analyses (bottom) of vimentin, N-cadherin, CD44, and E-cadherin levels in MDA-MB231cells treated with 0, 1, 2, $5 \mathrm{ng} / \mathrm{ml}$ of TGF- $\beta 1$ for $72 \mathrm{~h}$. GAPDH used as an internal control. $\mathbf{d}$ Real-time quantitative PCR (qPCR) analysis showed the mRNA level for GSN, E-cadherin, N-cadherin, and vimentin in MDA-MB231 cells treated with or without $2 \mathrm{ng} / \mathrm{ml}$ TGF- $\beta 1$ for 3 days. In $\mathbf{a}, \mathbf{b}, \mathbf{c}$, and $\mathbf{d}$, the values are the mean \pm SEM $(n=6)$, with * indicating a significant difference compared to the untreated cells 
Effects of GSN op on cell proliferation, cell cycle progression, and the expression of GSK-3 $\beta, \beta$-catenine, and cyclin D1 in MDA-MB231 and MCF-7 breast cancer cells

To further determine the functional role of increased GSN expression in the TGF- $\beta$ induced signaling for modulation of breast cancer cell progression, we conducted GSN overexpression (GSN op) in the two human breast cancer cell lines of MDA-MB231 and MCF-7. Stable clones of GSN op cells have 2- to 6- folds of GSN overexpression and the longer doubling time for cell proliferation in both MDA-MB231 and MCF-7 cells (Fig. 2a and c). This is consistent with the finding that TGF- $\beta 1$ treatment decreased cell proliferation (Fig. 1a) with increased GSN expression (Fig. 1b and d) in MDA-MB231 cells. Flow cytometry also revealed that cell cycle progression arrest at G0/G1 phase accompanying by halting cell cycle progression to DNA synthesis (S phase) occurred in GSN op MDA-MB231 and GSN op MCF-7 cells as compared to their controls, respectively (Fig. $2 \mathrm{~b}$ and $\mathrm{d}$ ).

Glycogen synthase kinase-3 3 (GSK-3 $\beta$ ) is a key component of multiple signaling pathways involved in the regulation of cell fate, protein synthesis, glycogen metabolism, cell mobility, proliferation, and survival [26-28]. By preventing cells from entering the cell cycle, GSK-3 $\beta$ participates in the regulation of the $\beta$-catenin signaling pathway by modulating cyclin D1 expression levels [29]. As compared to control cells, the expression levels of GSK-3 $\beta$ was also increased in concomitant with the decrease of the levels of cyclin D1 and $\beta$ catenin in GSN op MDA-MB231 and GSN op MCF-7 cells, which may cause the subsequent cell cycle arrest at the G1-S phase and hence halting DNA synthesis in those cells (Fig. 2e).

\section{Effects of GSN op on changes of cell morphology, cell surface elasticity, cytoskeletal protein expression, cell detachment, and migration in MDA-MB231 breast cancer cells}

In comparison with control MDA-MB 231 cells (Ctr), several GSN op clones of MDA-MB231 cells were found to alter morphological changes in cell shapes (Fig. 3a) with increased cell surface elasticity (Fig. $3 b$ ). Cell surface elasticity was determined by measuring adhesion force in the control and GSN op MDA-MB 231 cells (Fig. 3b). The adhesion force measured on the cell surface was $2.66 \pm 0.10$ and $3.30 \pm 0.13 \mathrm{nN}$ for the control and GSN op cells, respectively (Fig. 3b). Clearly, the upregulation of GSN could alter the cell surface adhesion associated with morphological modification in breast cancer cells. Since the dynamic formation of cell surface adhesion and detachment is required for cancer cell motility and invasion [30], we also determined the effect of GSN op on the cell detachment in breast cancer cells
(Fig. 3c). Result obtained showed that cell detachment from extracellular matrix was increased for GSN op cells as compared to control cells (Fig. 3c). This suggested that GSN severing the actin filament might contribute to offset the cell adhesion and or detachment to extracellular matrices in breast cancer cells. Interestingly, GSN op MDA-MB231 cells were also found to increase the protein content for Tropomyosin 1 (Tm1) as compared to controls (Fig. 3d). This is consistent with our previous finding that both GSN and Tm1 could affect the cell surface adhesion and cell proliferation in breast cancer cells [22]. To determine the effect of increased Tm1 expression levels on the cell detachment in GSN op MDA-MB231cells, siTm1 was conducted in the control and GSN op cells, respectively (Fig. 3e). Gene silencing Tm1 caused to decrease the cell detachment in both control and GSN op MDA-MB231 cells (Fig. 3e), suggesting that upregulation of Tm 1 by GSN op might contribute to facilitate cell detachment in MDA-MB231 cells. To verify that increases in GSN severing the actin filament caused to enhance cell motility in breast cancer cells, cell migration assay was compared in control and GSN op MDA-MB 231 cells (Fig. 3f). The result showed that GSN op significantly enhanced cell migration ( $~ 9$ fold) in MDA-MB231 cells (Fig. 3e). This is consistent with the previous finding that downregulation of GSN family proteins in MDA-MB 231 cells reduced the invasive and motile properties of breast cancer cells [31].

\section{Effects of GSN op and/or silencing by small interfering RNA on the expression of vimentin}

To confirm that GSN plays a crucial role in the TGF- $\beta 1$ induced EMT in breast cancer cells, studies with GSN op and/or siGSN were conducted in MDA-MB231 cells for measuring their effects on the expression levels of mesenchymal cell marker, vimentin (Fig. 4). Western analysis showed that in GSN op MDA-MB231 cells GSN op significantly increased the protein content of vimentin as compared to control cells without GSN op (Fig. 4a). The increase in the protein content of GSN and vimentin returned to the control level with siRNA treatment on GSN op cells (Top panel of Fig. 4a). In contrast, MDA-MB231 cells treated with siGSN caused decreases in the protein contents of vimentin and GSN by $\sim 86 \%$ and $\sim 42 \%$, respectively (Fig. 4 b).

TGF- $\beta 1$ induction increases the CD44+/CD24- subpopulation by coordinating gene expressions for CSC markers, EMT markers, and GSN in MDA-MB231 cells

To characterize CSC-like phenotypes in TGF- $\beta 1$ treated cells, fluorescence-activated cell sorting (FACS) flow cytometry was used to isolate subpopulation of CD44 +/CD24- for MDA-MB-231 cells. Under the condition 


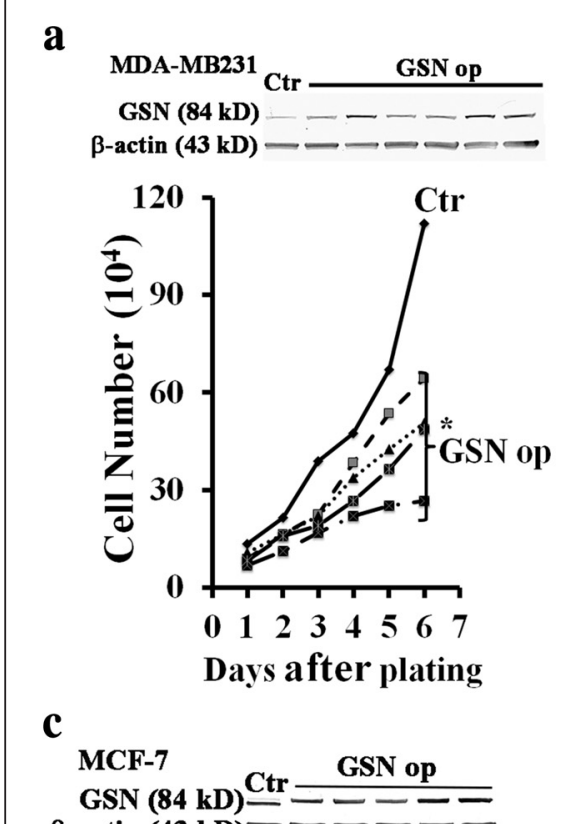

b

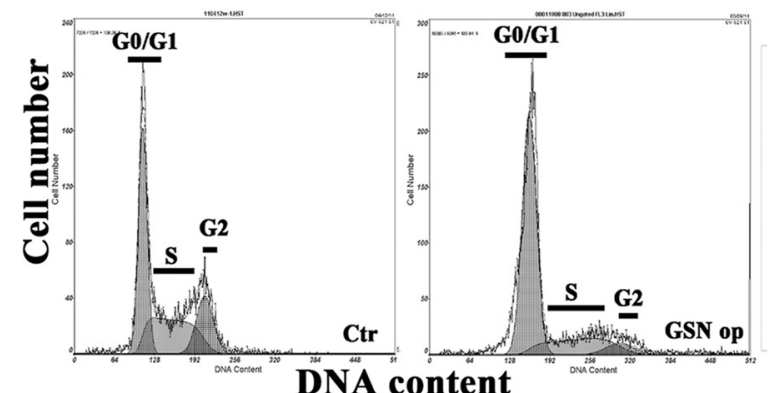

DNA content

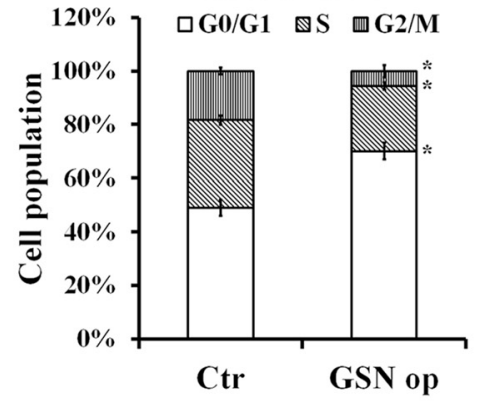

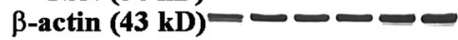

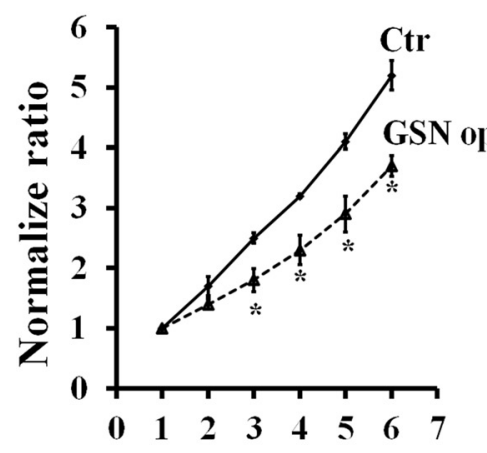

Days after plating

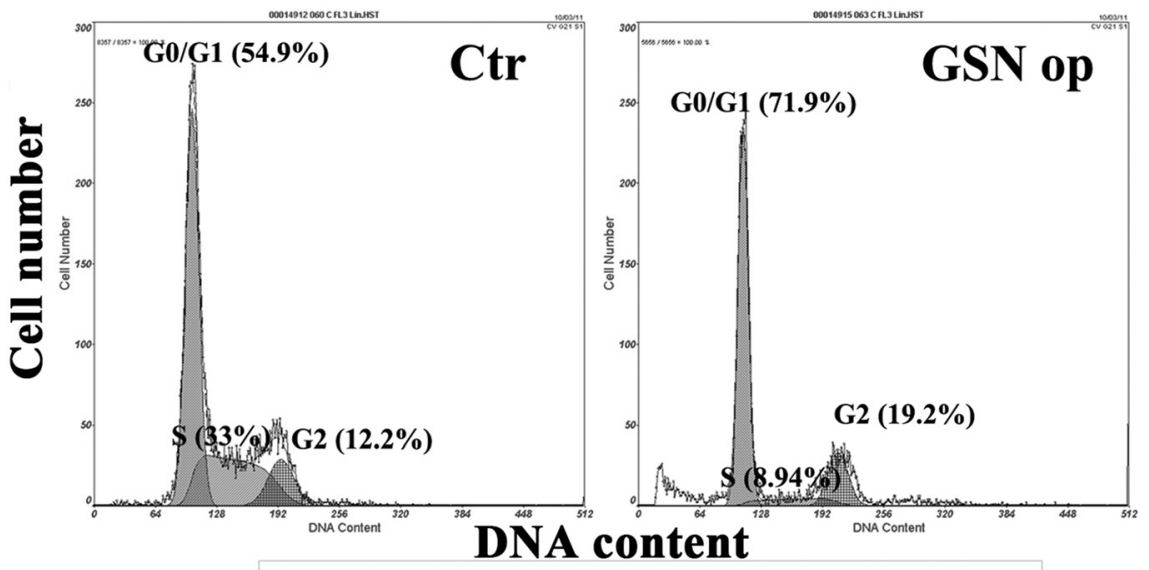

$\mathbf{e}$
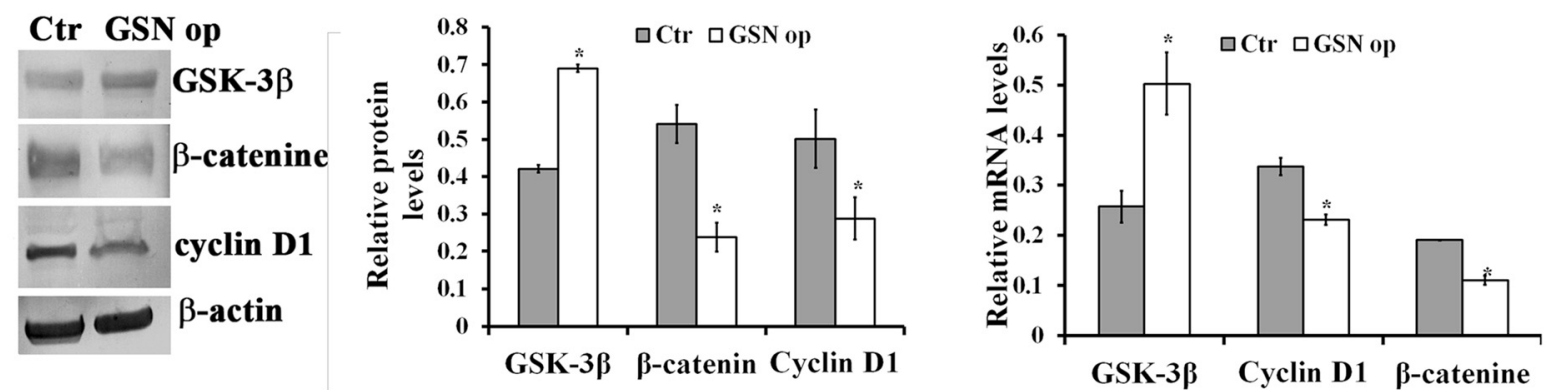

Fig. 2 Effects of GSN op on cell proliferation, cell cycle progression, and the expression of GSK-3 $\beta$, $\beta$-catenine, and cyclin D1 in MDA-MB231 and MCF-7 breast cancer cells. GSN overexpression increased the doubling time for cell proliferation in stable GSN-overexpressing (GSN op) clones of (a) MDA-MB231, and (c) MCF-7 breast cancer cells as compared to their controls (Ctr), respectively. Flow cytometry determined the cell population at different cell cycle phases: G0/G1, S, and G2 in control and GSN op cells of (b) MDA-MB231, and (d) MCF-7 cells, respectively. e GSN overexpression altered the protein levels and mRNA expression of GSK-3ß, $\beta$-catenin, cyclin D1 in MDA-MB231 or MCF-7 cells. $\beta$-actin used as an internal control. In $\mathbf{a}, \mathbf{b}, \mathbf{c}, \mathbf{d}$ and $\mathbf{e}$, the values are the mean $\pm \operatorname{SEM}(n=6)$, with * indicating a significant difference compared to the cells in control and GSN op, respectively 


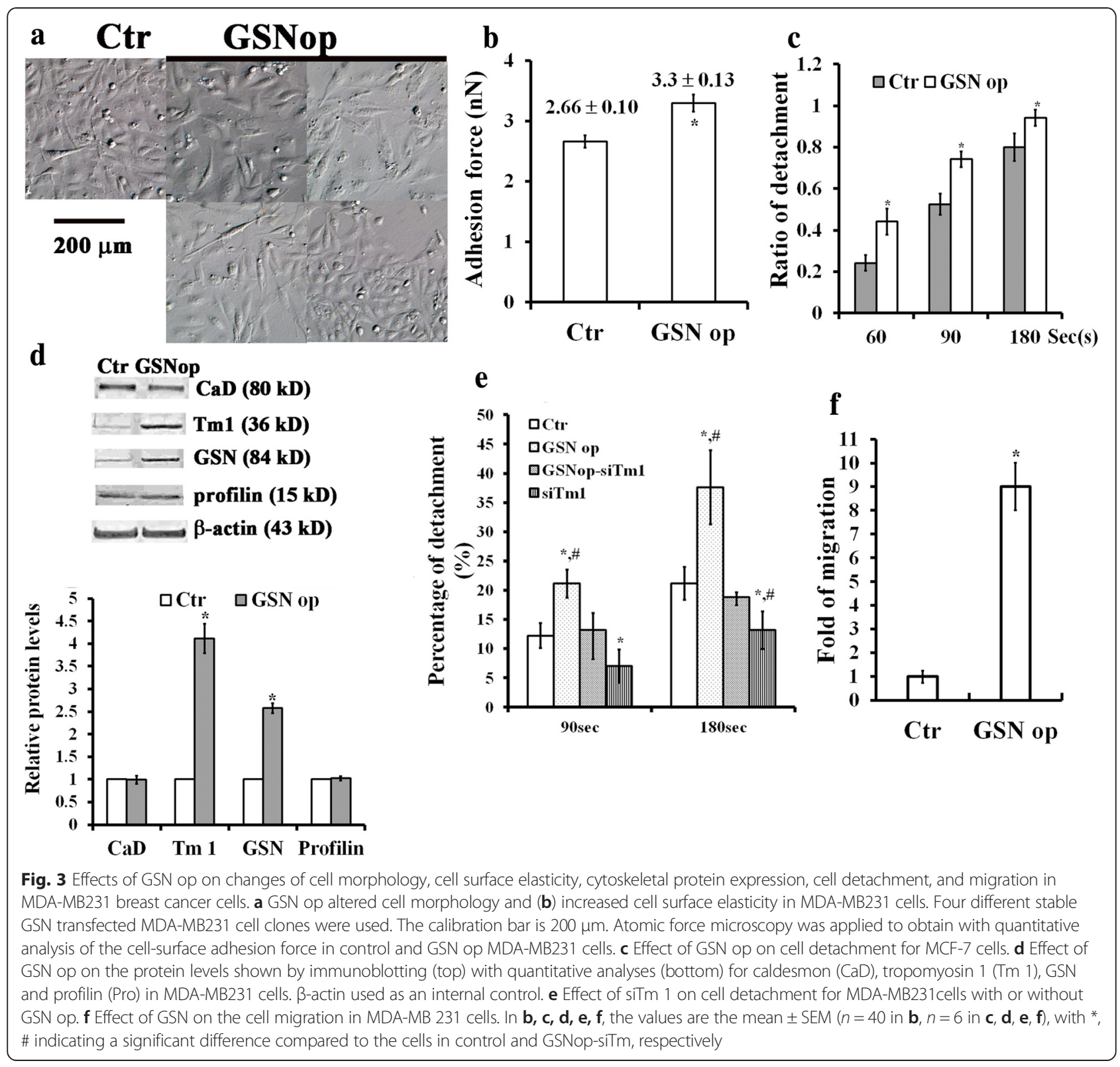

of $2 \mathrm{ng} / \mathrm{ml}$ TGF- $\beta 1$ for 3 days, the population of CD44 +/CD24- MDA-MB231 breast cancer cells were increased (Fig. 5a). After collection by FACS flow cytometry, these cells were found to increase the gene markers for stem cell pluripotency (i.e. Oct4, Sox2 and Nanog) (Fig. 5b), the gene expression for mesenchymal cell markers such as $\mathrm{N}$-cadherin, and vimentin, but to decrease in the gene expression for epithelial cell marker such as E-cadherin (Fig. 5c). This result indicated that TGF- $\beta 1$ increases stem cell function and EMT in the CD44+/CD24- subpopulation of MDAMB231 breast cancer cells. In addition, we also found that TGF- $\beta 1$ increased the expression of GSN in the CD44+/CD24- MDA-MB231 cells (Fig. 5d).
TGF- $\beta 1$ induced epigenetic regulation of GSN gene expressions in the CD44+/CD24- subpopulation of MDA-MB231 cells

Alterations in GSN RNA expression in most breast cancers of rats, mice, and humans have been shown not due to gross mutations of the GSN gene [30]. Alternately, another route to modulate GSN expression is via epigenetic modification on GSN gene promotor [32-34]. To test whether the TGF- $\beta 1$ causes the epigenetic modification on GSN expression in breast cancer cells, the method of methylation-specific PCR (MSPCR) for assessing the methylation and unmethylation on the $\mathrm{CpG}$ island at the promoter region of GSN (intron 1) was used in MDA-MB231 cells without FACS sorting (control), in 


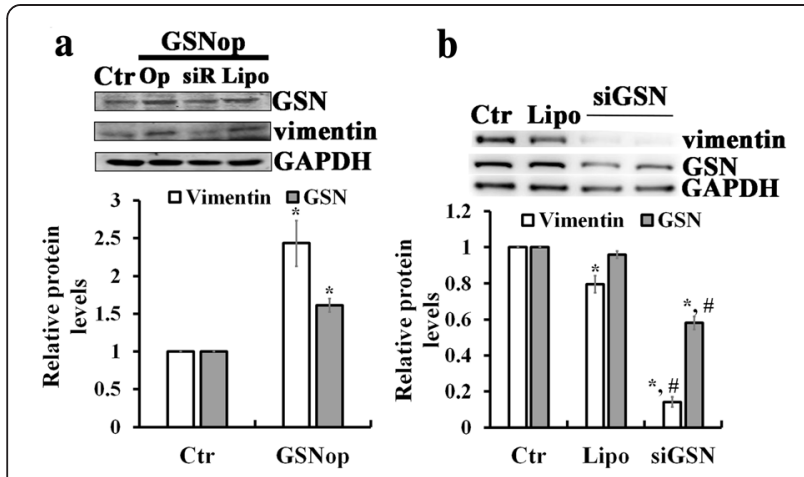

Fig. 4 Effects of GSN op and/or silencing by small interfering RNA on the expression of vimentin in MDA-MB231 breast cancer cells. a Western blotting (top) with quantitative analyses (bottom) of GSN, and vimentin, levels in control MDA-MB231 cells (Ctr), and GSN op MDA-MB231cells treated with and/ or without siRNA against GSN (siR) or lipofemitamine (Lipo). $\mathbf{b}$ Effect of siGSN on the protein content of vimentin, and GSN in MDA-MB231 cells (Ctr). GAPDH used as an internal control. In $\mathbf{a}$ and $\mathbf{b}$, the values are the mean \pm SEM $(n=3)$, with ${ }^{*}$, \# indicating a significant difference compared to the control cells and Lipo-treated cells, respectively
CD44+/CD22- subpopulation sorted cells without TGF$\beta 1$ pretreatment, and in $\mathrm{CD} 44+/ \mathrm{CD} 22$ - subpopulation sorted cells with $2 \mathrm{ng} / \mathrm{ml}$ TGF- $\beta 1$ pretreatment for 3 days (Fig. 6a). In control MDA-MB231 cells without TGF- $\beta 1$ stimulation, CD44+/CD22- subpopulation sorting cells increased methylation by 10 folds but decreased unmethylation by $68 \%$. Interestingly, TGF- $\beta 1$ pretreatment reduced $48 \%$ methylation but increased 4 fold unmethylation of GSN promotor in the CD44+/CD22- subpopulation sorted cells (Fig. 6a). Consistently, two major DNA methyltransferases, DNMT 1 and DNMT 3B, were found to decrease their expression by 45 and $49 \%$, respectively, with TGF- $\beta 1$ pretreatment in the CD44+/CD22 subpopulation sorted MDA-MB231 cells (Fig. 6b).

\section{Discussion}

A subpopulation of cancer stem cells (CSCs) within heterogeneous metastatic breast tumors have the ability to differentiate into all the different cells found within a tumor and they have stem cell characteristics, including self-renewal, pluripotency, motility, tumor recurrence, and

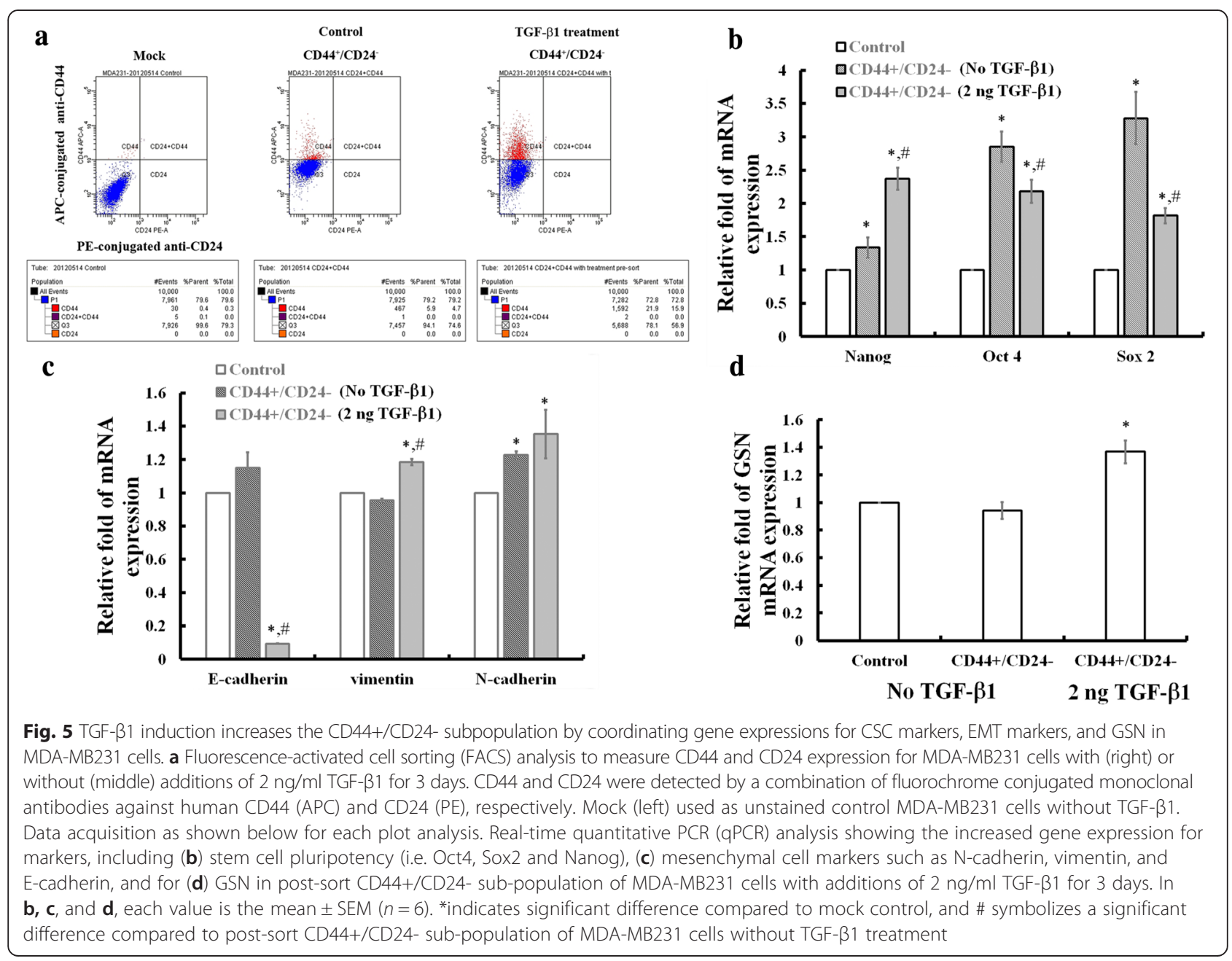




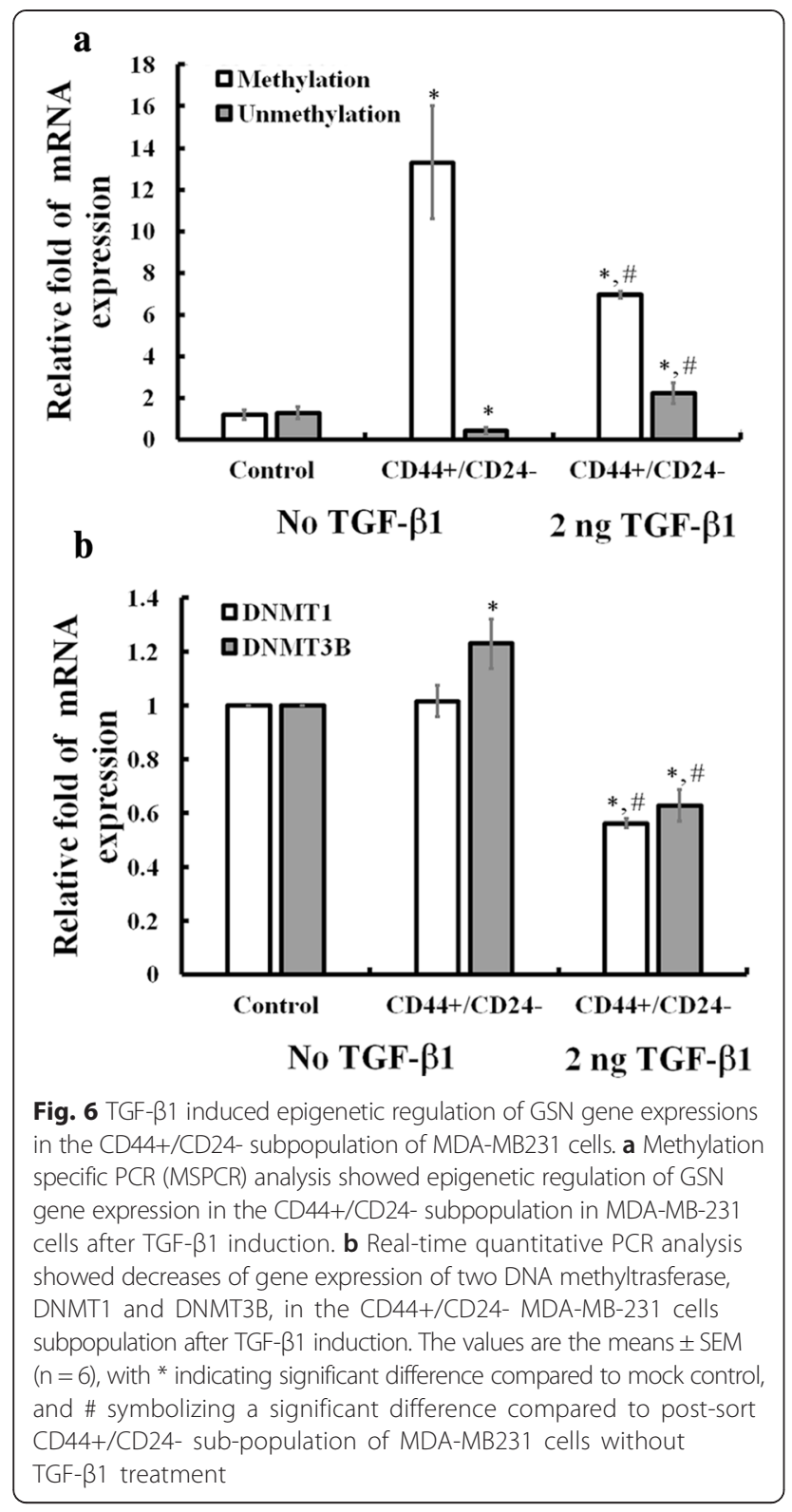

chemotherapy-resistance [35-37]. By sorting a subpopulation of CD44+/CD24-(low) cells from human breast cancer tissue, Al-Hajj et al. were the first to demonstrate that these cells can be enriched for breast CSCs and to develop a tumor in immune-deficient mice [18]. In addition, TGF$\beta$ has been shown to increase CSC numbers by producing gene markers linked to stem cell function and Epithelial to Mesenchymal Transition (EMT) in breast cancers [6]. More recently, evidence also showed that TGF- $\beta$ could increase breast CSCs in the low claudin subtype of breast tumors [5]. In this study, we verified that with TGF- $\beta 1$ treatment for 3 days the gene expression for CD44 and GSN was increased in MDA-MB231 cells (Fig. 1) with increasing the gene expression of EMT markers (Fig. 1) for enhancing their cell migration and invasion. Our study with FACS-flow cytometry also confirmed that TGF- $\beta 1$ induction increased the CD44+/CD24- subpopulation of MDA-MB231 cells (Fig. 5a). In the TGF- $\beta 1$ enriched CD44+/CD24- cells the mRNA expression levels for the markers of stem cell pluripotency (i.e. Oct4, Sox2 and Nanog) (Fig. 5b) were found to be increased in concomitance with the increased expression for mesenchymal cell markers (i.e. N-cadherin, Vimentin) but the decreased expression for epithelial cell marker (i.e. E-cadherin) (Fig. 5c). It is of note that the GSN expression level is higher for the TGF- $\beta 1$ induction than for without TGF- $\beta 1$ treatment (Fig. 5d) in the CD44+/CD24- subpopulation of MDAMB231 cells. To test whether GSN plays a role in controlling cell proliferation and motility, we conducted GSN overexpression (GSN op) in the two human breast cancer cell lines of MDA-MB231 and MCF-7 (Figs. 2, 3). The results showed that GSN op altered cell morphology (Fig. 2) and increased cell surface elasticity with an increase in cell detachment, by which cause to increase the cell migration/invasion (Fig. 3). In addition, we also verified that GSN plays a role in the gene expression for the mesenchymal cell marker, vimentin in breast cancer cells with GSN op and/or siGSN approaches (Fig. 4). Taken together, the present study suggested that the modification of GSN expression might involve in the TGF- $\beta 1$ signaling events for inducing cancer cell stemness and increasing cell migration and invasion in $\mathrm{CD} 44+/ \mathrm{CD} 24-$ subpopulation of breast cancer cells.

The regulation of GSN expression is varied in many different tumors [38-44]. In oral cancers, biphasic expression of GSN was found during the progression of carcinogenesis [40, 41]. Decreased GSN expression has been found in many transformed and malignant cancer cells, including breast cancers [42-44]. Evidence indicated that GSN gene loss is one of the most common disorders in invasive and metastatic breast cancers [45, 46]. Studies have shown that $71 \%$ of human sporadic, invasive breast carcinomas and $56 \%$ of ductal carcinomas in situ were strikingly deficient in the GSN protein $[45,46]$. The clinical evidence also indicated that the GSN expression may be associated with survival from malignant breast cancers, and the frequency of GSN deficiency increases significantly with progression to invasive phenotypic cancer cells [45]. Recent studies have found the increased GSN expressions in chemo-resistant head and-neck (HNC) [47] and gynecological cancers [48]. These studies suggested that GSN might play important roles for chemoresistance in cancers. Interestingly, the present study showed that increased GSN expression is associated with the TGF- $\beta 1$ signaling for breast CSC differentiation. Different cell populations of breast cancer cells vary their GSN expression in response to TGF- $\beta 1$ induction (Fig. $5 \mathrm{~d}$ ). Only CSC-like cells (i.e. CD44+/CD24-) in breast cancer cells respond 
to TGF- $\beta 1$ induction for increasing in GSN expression such as to maintain their invasive phenotype. It will be of interest to verify whether the TGF- $\beta 1$ modified GSN expression is involved in chemoresistance in breast cancers.

Evidence showed that the GSN down-regulation is due to decreased activity of the GSN promoter by activating transcription factor-1 [49]. It was suggested that GSN expression and function can be further influenced by epigenetic changes [16]. Epigenetic modulation involves modifications of the transcriptional activation of certain genes [33, 34]. In the present study, we showed that the $\mathrm{CpG}$ island methylation of the GSN gene was decreased in CD44+/CD24population of MDA-MB231 cells after TGF- $\beta 1$ induction as compared to cells without treatment (Fig. 6a). Accordingly, TGF- $\beta 1$ increased GSN gene expression in $\mathrm{CD} 44+/ \mathrm{CD} 24-$ population through decreases of DNA methylation of CpG island at GSN promotor by inhibition of two major DNA methyltransferase, DNMT1 and DNMT3B. These two DNA methyltransferase were decreased in the CD44+/CD24- subpopulation of MDAMB231 cells after TGF- $\beta 1$ treatment (Fig. 6b). Apparently, TGF- $\beta 1$ induction attenuated the methylation but facilitated the unmethylation on GSN promoter region in breast cancer cells such as to remove the inhibition on GSN gene expression in MDA-MB231 cancer cells. Such information on the relationship between TGF- $\beta 1$ and its control on DNA methyltransferase-dependent gene expression may have an important impact on the clinical therapy of patients with metastatic breast cancers.

\section{Conclusion}

Our results suggested that TGF- $\beta 1$ acting by epigenetic modulation of GSN gene expression might be linked to the signalling events for breast cancer stem cell differentiation.

\section{Competing interests}

The authors declare that they have no competing interests.

\section{Authors' contributions}

CZY performed experiments for testing TGF-b1 effects on the increases in GSN expression, EMT, and cancer cell stemness in MDA-MB231 cells. WPW participated in FACS, cell migration/invasion assay. SDB participated in constructing the pc6-GSN plasmid, MSPCR analysis, as well as the design of the study. CKY conducted GSN overexpression in MDA-MB231 and MCF-7 cells, and atomic force microscopy for measuring cell surface elasticity. LYM conceived of the study and participated in its design and coordination and helped to draft the manuscript and final MS submission. All authors read and approved the final manuscript.

\section{Acknowledgements}

This work was supported by the National Science Council of Taiwan government (to Y-M L., Grant: NSC 102-2320-B-005-002). The authors also wished to thank Ms Meng-Shiun Chang for performing the experiments on migration assay in GSN op MDA-MB231 cells.

\section{Author details}

'Department of Life Sciences, National Chung-Hsing University, Taichung 40227, Taiwan. ${ }^{2}$ Institute of Basic Medical Sciences, National Cheng Kung University, Tainan 701, Taiwan. ${ }^{3}$ Institute of Oral Medicine, National Cheng Kung University, Tainan 701, Taiwan. ${ }^{4}$ Rong Hsing Research Center for
Translational Medicine, National Chung Hsing University, Taichung 40227, Taiwan.

Received: 3 July 2015 Accepted: 29 September 2015

Published online: 20 October 2015

\section{References}

1. Massagué J. TGFß signalling in context. Nat Rev Mol Cell Biol. 2012;13:616-30.

2. Roberts $A B$, Wakefield $L M$. The two faces of transforming growth factor beta in carcinogenesis. Proc Natl Acad Sci U S A. 2003;100:8621-3.

3. Tian M, Neil JR, Schiemann WP. Transforming growth factor- $\beta$ and the hallmarks of cancer. Cell Signal. 2011;23:951-62.

4. Schmierer B, Hill CS. TGFbeta-SMAD signal transduction: molecular specificity and functional flexibility. Nat Rev Mol Cell Biol. 2007;8:970-82.

5. Bruna A, Greenwood W, Le Quesne J, Teschendorff A, Miranda-Saavedra D, Rueda OM, et al. TGF $\beta$ induces the formation of tumour-initiating cells in claudinlow breast cancer. Nat Commun. 2012;3:1055.

6. Tan AR, Alexe G, Reiss M. Transforming growth factor-beta signaling: emerging stem cell target in metastatic breast cancer? Breast Cancer Res Treat. 2009;115:453-95.

7. Olson MF, Sahai E. The actin cytoskeleton in cancer cell motility. Clin Exp Metastasis. 2009:26:273-87.

8. Chhabra ES, Higgs HN. The many faces of actin: matching assembly factors with cellular structures. Nat Cell Biol. 2007:9:1110-21.

9. Bursac P, Lenormand G, Fabry B, Oliver M, Weitz DA, Viasnoff V, et al. Cytoskeletal remodelling and slow dynamics in the living cell. Nat Mater 2005;4:557-61.

10. Lambrechts A, Van Troys M, Ampe C. The actin cytoskeleton in normal and pathological cell motility. Int J Biochem Cell Biol. 2004;36:1890-909.

11. Bakin AV, Safina A, Rinehart C, Daroqui C, Darbary H, Helfman DM. A critical role of tropomyosins in TGF-beta regulation of the actin cytoskeleton and cell motility in epithelial cells. Mol Biol Cell. 2009;15:4682-94.

12. Edlund S, Landström M, Heldin CH, Aspenström P. Transforming growth factor-beta-induced mobilization of actin cytoskeleton requires signaling by small GTPases Cdc42 and RhoA. Mol Biol Cell. 2002;13:902-14.

13. Kiselar JG, Janmey PA, Almo SC, Chance MR. Visualizing the $\mathrm{Ca}^{2+}$-dependent activation of gelsolin by using synchrotron footprinting. Proc Natl Acad Sci U S A. 2003;100:3942-7.

14. Yin HL, Stossel TP. Control of cytoplasmic actin gel-sol transformation by gelsolin, a calcium-dependent regulatory protein. Nature. 1979;281:583-6.

15. Marino N, Marshall JC, Collins JW, Zhou M, Qian Y, Veenstra T, et al. Nm23-h1 binds to gelsolin and inactivates its actin-severing capacity to promote tumor cell motility and metastasis. Cancer Res. 2013;73:5949-62.

16. Li GH, Arora PD, Chen Y, McCulloch CA, Liu P. Multifunctional roles of gelsolin in health and diseases. Med Res Rev. 2012;32:999-1025.

17. Kwiatkowski DJ. Functions of gelsolin: motility, signaling, apoptosis, cancer. Curr Opin Cell Biol. 1999;11:103-8.

18. Al-Hajj M, Wicha MS, Benito-Hernandez A, Morrison SJ, Clarke MF. Prospective identification of tumorigenic breast cancer cells. Proc Natl Acad Sci U S A. 2003;100:3983-8.

19. Hwang-Verslues WW, Kuo WH, Chang PH, Pan CC, Wang HH, Tsai ST, et al. Multiple lineages of human breast cancer stem/progenitor cells identified by profiling with stem cell markers. PLoS ONE. 2009;4:e8377.

20. Giatromanolaki A, Sivridis E, Fiska A, Koukourakis MI. The CD44+/CD24phenotype relates to 'triple-negative' state and unfavorable prognosis in breast cancer patients. Med Oncol. 2011;28:745-52.

21. Hennessy BT, Gonzalez-Angulo AM, Stemke-Hale K, Gilcrease MZ, Krishnamurthy S, Lee JS, et al. Characterization of a naturally occurring breast cancer subset enriched in epithelial-to-mesenchymal transition and stem cell characteristics. Cancer Res. 2009;69:4116-24.

22. Hsu YC, Liou YM. The anti-cancer effects of (-)-epigalocathine-3-gallate on the signaling pathways associated with membrane receptors in MCF-7 cells. J Cell Physiol. 2011;226:2721-30.

23. Shieh DB, Li RY, Liao JM, Chen GD, Liou YM. Effects of genistein on $\beta$-catenin signaling and subcellular distribution of actin-binding proteins in human umbilical CD105-positive stromal cells. J Cell Physiol. 2010;223:423-34.

24. Peng KW, Liou YM. Differential role of actin-binding proteins in controlling the adipogenic differentiation of human CD105-positive Wharton's Jelly cells. Biochim Biophys Acta. 1820;2012:469-81.

25. Kwiatkowski DJ, Mehl R, Yin HL. Genomic organization and biosynthesis of secreted and cytoplasmic forms of gelsolin. J Cell Biol. 1988;106:375-84. 
26. Gupta C, Kaur J, Tikoo K. Regulation of MDA-MB-231 cell proliferation by GSK-3 $\beta$ involves epigenetic modifications under high glucose conditions. Exp Cell Res. 2014;324:75-83.

27. Jacobs KM, Bhave SR, Ferraro DJ, Jaboin JJ, Hallahan DE, Thotala D. GSK-3ß: A Bifunctional Role in Cell Death Pathways. Int J Cell Biol. 2012;2012:930710.

28. Luo J. Glycogen synthase kinase 3beta (GSK3beta) in tumorigenesis and cancer chemotherapy. Cancer Lett. 2009;273:194-200.

29. Takahashi-Yanaga F, Sasaguri T. GSK-3beta regulates cyclin D1 expression: a new target for chemotherapy. Cell Signal. 2008;20:581-9.

30. Yin S, Lockett J, Meng Y, Biliran Jr H, Blouse GE, Li X, et al. Maspin retards cell detachment via a novel interaction with the urokinase-type plasminogen activator/urokinase-type plasminogen activator receptor system. Cancer Res. 2006;66:4173-81.

31. Van den Abbeele A, De Corte V, Van Impe K, Bruyneel E, Boucherie C, Bracke $M$, et al. Downregulation of gelsolin family proteins counteracts cancer cell invasion in vitro. Cancer Lett. 2007;255:57-70.

32. Mielnicki LM, Ying AM, Head KL, Asch HL, Asch BB. Epigenetic Regulation of Gelsolin Expression in Human Breast Cancer Cells. Exp Cell Res. 1999;249:161-76.

33. Haga K, Fujita H, Nomoto M, Sazawa A, Nakagawa K, Harabayashi T, et al. Gelsolin gene silencing involving unusual hypersensitivities to dimethylsulfate and $\mathrm{KMnO} 4$ in vivo footprinting on its promoter region. Int J Cancer. 2004;111:873-80.

34. Zhang Y, Fatima N, Dufau ML. Coordinated changes in DNA methylation and histone modifications regulate silencing/derepression of luteinizing hormone receptor gene transcription. Mol Cell Biol. 2005;25:7929-39.

35. Luo M, Brooks M, Wicha MS. Epithelial-mesenchymal plasticity of breast cancer stem cells: implications for metastasis and therapeutic resistance. Curr Pharm Des. 2015;21:1301-10.

36. Pinto CA, Widodo E, Waltham M, Thompson EW. Breast cancer stem cells and epithelial mesenchymal plasticity - Implications for chemoresistance. Cancer Lett. 2013;341:56-62.

37. Velasco-Velázquez MA, Popov VM, Lisanti MP, Pestell RG. The role of breast cancer stem cells in metastasis and therapeutic implications. Am J Pathol. 2011;179:2-11.

38. Litwin M, Nowak D, Mazur AJ, Baczyńska D, Mannherz HG, Malicka-Błaszkiewicz M. Gelsolin affects the migratory ability of human colon adenocarcinoma and melanoma cells. Life Sci. 2012;90:851-61.

39. Radwanska A, Litwin M, Nowak D, Baczynska D, Wegrowski Y, Maquart FX, et al. Overexpression of lumican affects the migration of human colon cancer cells through up-regulation of gelsolin and filamentous actin reorganization. Exp Cell Res. 2012;318:2312-23.

40. Deng R, Hao J, Han W, Ni Y, Huang X, Hu Q. Gelsolin regulates proliferation, apoptosis, migration and invasion in human oral carcinoma cells. Oncol Lett. 2015;9:2129-34.

41. Shieh DB, Chen IW, Wei TY, Shao CY, Chang HJ, Chung CH, et al. Tissue expression of gelsolin in oral carcinogenesis progression and its clinicopathological implications. Oral Oncol. 2006:42:599-606.

42. Liu J, Liu YG, Huang R, Yao C, Li S, Yang W, et al. Concurrent downregulation of Egr-1 and gelsolin in the majority of human breast cancer cells. Cancer Genomics Proteomics. 2007;4:377-85.

43. Thor AD, Edgerton SM, Liu S, Moore 2nd DH, Kwiatkowski DJ. Gelsolin as a negative prognostic factor and effector of motility in erbB-2-positive epidermal growth factor receptor-positive breast cancers. Clin Cancer Res. 2001;7:2415-24.

44. Winston JS, Asch HL, Zhang PJ, Edge SB, Hyland A, Asch BB. Downregulation of gelsolin correlates with the progression to breast carcinoma. Breast Cancer Res Treat. 2001;65:11-21.

45. Asch HL, Winston JS, Edge SB, Stomper PC, Asch BB. Down-regulation of gelsolin expression in human breast ductal carcinoma in situ with and without invasion. Breast Cancer Res Treat. 1999;55:179-88.

46. Asch HL, Head K, Dong Y, Natoli F, Winston JS, Connolly JL, et al. Widespread loss of gelsolin in breast cancers of humans, mice, and rats. Cancer Res. 1996;56:4841-5.

47. Wang PW, Abedini MR, Yang LX, Ding AA, Figeys D, Chang JY, et al. Gelsolin regulates cisplatin sensitivity in human head-and-neck cancer. Int J Cancer. 2014;135:2760-9.

48. Abedini MR, Wang PW, Huang YF, Cao M, Chou CY, Shieh DB, et al. Cell fate regulation by gelsolin in human gynecologic cancers. Proc Natl Acad Sci U S A. 2014;111:14442-7.

49. Dong Y, Asch HL, Ying A, Asch BB. Molecular mechanism of transcriptional repression of gelsolin in human breast cancer cells. Exp Cell Res. 2002;276:328-36.

\section{Submit your next manuscript to BioMed Central and take full advantage of:}

- Convenient online submission

- Thorough peer review

- No space constraints or color figure charges

- Immediate publication on acceptance

- Inclusion in PubMed, CAS, Scopus and Google Scholar

- Research which is freely available for redistribution

Submit your manuscript at www.biomedcentral.com/submit 